Proyecciones Journal of Mathematics

Vol. 33, $\mathrm{N}^{\circ}$ 4, pp. 447-470, December 2014.

Universidad Católica del Norte

Antofagasta - Chile

\title{
Subseries convergence in abstract duality pairs
}

\author{
Min-Hyung Cho \\ Kum-Oh national Institute of Tech., Korea \\ Li Ronglu \\ Harbin Institute of Tech., P. R. C. \\ and \\ Charles Swartz \\ New Mexico State University, U.S.A. \\ Received: October 2014. Accepted : October 2014
}

\begin{abstract}
Let $E, F$ be sets, $G$ an Abelian topological group and $b: E \times F \rightarrow G$. Then $(E, F, G)$ is called an abstract triple. Let $w(F, E)$ be the weakest toplogy on $F$ such that the maps $\{b(x, \cdot): x \in E\}$ from $F$ into $G$ are continuous. A subset $B \subset F$ is $w(F, E)$ sequentially conditionally compact if every sequence $\left\{y_{k}\right\} \subset B$ has a subsequence $\left\{y_{n_{k}}\right\}$ such that $\lim _{k} b\left(x, y_{n_{k}}\right)$ exists for every $x \in E$. It is shown that if a formal series $\sum x_{j}$ in $E$ is subseries convergent in the sense that for every subsequence $\left\{x_{n_{j}}\right\}$ there is an element $x \in E$ such that $\sum_{j=1}^{\infty} b\left(x_{n_{j}}, y\right)=$ $b(x, y)$ for every $y \in F$, then the series $\sum_{j=1}^{\infty} b\left(x_{n_{j}}, y\right)$ converge uniformly for $y$ belonging to $w(F, E)$ sequentially conditionally compact subsets of F. This result is used to establish Orlicz-Pettis Theorems in locall convex and function spaces. Applications are also given to Uniform Boundedness Principles and continuity results for bilinear mappings.
\end{abstract}


In this paper we give an abstraction of the classical notion of duality between two vector spaces and prove several versions of the Orlicz-Pettis Theorem for subseries convergent series in the abstract setting. We give a number of applications to classical versions of the Orlicz-Pettis Theorem as well as versions of the theorem in various function spaces and spaces of operators, the Uniform Boundedness Priciple and a version of the MazurOrlicz Theorem on continuity of bilinear operators.

The notion of abstract triples was first developed in the mathematics department of New Mexico State University in the period of 1988-1990 when Professor Ronglu Li was a visiting scholar from China. After returning to China, Li invited Min-Hyung Cho to join in the development of the subject. The original notes were never published in full although various results have appeared in publications (see the remarks following Example 8). This paper contains a full development of the original results with some improvements and additional results which were obtained later; see in particular the Uniform Boundedness Principle and the Mazur-Orlicz results in Cases 29, 31 and 32.

This paper is dedicated to the memory of Professor Ronglu Li who tragically passed away on Feb. 4, 2014.

\begin{abstract}
Triples.
Let $E, F$ be sets and $G$ a Hausdorff, abelian topological group with $b: E \times F \rightarrow G$; if $x \in E$ and $y \in F$, we often write $b(x, y)=x \cdot y$ for convenience. We refer to $E, F, G$ as an abstract duality pair with respect to $G$ or an abstract triple and denote this by $(E, F: G)$. In what follows $(E, F: G)$ will denote an abstract triple. Note that $(F, E: G)$ is an abstract triple under the map $\bar{b}(y, x)=b(x, y)$.

We give examples of abstract triples shortly.

Let $w(E, F)$ be the weakest topology on $E$ such that the family of maps $\{b(\cdot, y): y \in F\}$ are continuous from $E$ into $G$.

If $\sum g_{j}$ is a formal series in $G$, the series is subseries convergent if the series $\sum_{j=1}^{\infty} g_{n_{j}}$ converges in $G$ for every subsequence $\left\{n_{j}\right\}$. If $\sigma$ is an infinite subset of $\mathbf{N}$, we write $\sum_{j \in \sigma} g_{j}=\sum_{j=1}^{\infty} g_{n_{j}}$, where the elements of $\sigma$ are arranged in a subsequence $\left\{n_{j}\right\}$; if $\sigma$ is finite, the meaning of $\sum_{j \in \sigma} g_{j}$ is clear.
\end{abstract}

Definition 1. A sequence $\left\{x_{j}\right\} \subset E$ or a (formal) series $\sum x_{j}$ is $w(E, F)$ 
subseries convergent if for every $\sigma \subset \mathbf{N}$, there exists $x_{\sigma} \in E$ such that

$$
\sum_{j \in \sigma} x_{j} \cdot y=x_{\sigma} \cdot y
$$

for every $y \in F$. We symbolically write $\sum_{j \in \sigma} x_{j}=x_{\sigma}$ and say that the series $\sum x_{j}$ is $w(E, F)$ subseries convergent.

Note that we do not assume any algebraic structure on $E$, the algebraic operations are transferred to $G$ via the map $b: E \times F \rightarrow G$; of course, if the set $E$ has sums defined on it the meaning of $\sum_{j \in \sigma} x_{j}$ is clear.

We give some examples which will be employed later.

Example 2. Of course, the simplest example of an abstract triple is a pair of vector spaces $E, F$ in duality where $G$ is just the scalar field and the topology $w(E, F)$ is just the weak topology $\sigma(E, F)$ from the duality. In this case, if $\sum x_{j}$ is $w(E, F)$ subseries convergent, $\sum_{j \in \sigma} x_{j}$ is the usual weak sum.

Example 3. Let $\Sigma$ be a $\sigma$-algebra of subsets of a set $S$ and $\mathcal{M}$ be a family of countably additive $G$ valued measures on $\Sigma$. Define $b: \Sigma \times \mathcal{M} \rightarrow$ $G$ by $b(A, \mu)=\mu(A)$ so $(\Sigma, \mathcal{M}: G)$ is an abstract triple. If $\left\{A_{j}\right\}$ is a pairwise disjoint sequence from $\Sigma$, then the series $\sum A_{j}$ is $w(\Sigma, \mathcal{M})$ subseries convergent with $\sum_{j \in \sigma} A_{j}=\cup_{j \in \sigma} A_{j}$.

Example 4. Let $E, F$ be abelian groups such that there exists a biadditive map $b: E \times F \rightarrow G$. Then $(E, F: G)$ is an abstract triple; this abstract setting was utilized in [Sw2].

Example 5. Let $E$ be a topological space and $C(E, G)$ be the space of all continuous maps from $E$ into $G$. If $b(x, f)=f(x)$ for $x \in E$ and $f \in$ $C(E, G)$, then $(E, C(E, G): G)$ is an abstract triple. Also, $(C(E, G), E: G)$ is an abstract triple under the map $(f, x) \rightarrow f(x)$.

Example 6. Let $E, G$ be topological vector spaces and $L(E, G)$ the space of all continuous linear operators from $E$ into $G$, then $(L(E, G), E: G)$ forms an abstract triple under the map $b(T, x)=T(x)$; in this case the topology $w(L(E, G), E)$ is just the strong operator topology. Also, $(E, L(E, G)$ : $G)$ forms an abstract triple under the map $b(x, T)=T(x)$. 
Example 7. Let $X$ be a Hausdorff topological vector space and $E$ be a vector space of $X$ valued sequences which contains the subspace $c_{00}(X)$ of all $X$ valued sequences which are eventually 0 . Assume that $E$ has a vector topology under which it is an $A K$ space,i.e., the coordinate projection $P_{k}$ which sends each sequence $x=\left(x_{1}, x_{2}, \ldots\right)$ in $E$ into the sequence with $x_{k}$ in the $k^{\text {th }}$ coordinate and 0 in the other coordinates is continuous and if $Q_{k}=\sum_{j=1}^{k} P_{j}$, then $Q_{k} x \rightarrow x$ in the topology of $E$ for every $x \in E$. Let $F=\left\{Q_{k}: k \in \mathbf{N}\right\}$. Then $(E, F: E)$ is an abstract triple under the map $\left(x, Q_{k}\right)=Q_{k} x$. This situation covers the case of the sequence spaces $l^{p}(X)$ and $c_{0}(X)$ when $X$ is a locally convex space.

Example 8. Let $X$ be a Hausdorff topological vector space and $E$ be a vector space of $X$ valued sequences which contains the subspace $c_{00}(X)$ of all $X$ valued sequences which are eventually 0 . Let $Y$ be a topological vector space. The $\beta$-dual of $E$ with respect to $Y$ is defined to be

$$
E^{\beta Y}=\left\{\left\{T_{j}\right\} \subset L(X, Y): \sum_{j=1}^{\infty} T_{j} x_{j} \text { converges for every } x=\left\{x_{j}\right\} \in E\right\} .
$$

Then $\left(E, E^{\beta Y}: Y\right)$ is an abstract triple under the map $\left(\left\{x_{j}\right\},\left\{T_{j}\right\}\right) \rightarrow$ $\sum_{j=1}^{\infty} T_{j} x_{j}$.

Other examples will be given in the application section.

There have been several similar abstractions of the duality between vector spaces which have been used to treat versions of the Orlicz-Pettis Theorem. For example, Blasco, Calabuig and Signes ([BCS]) have considered a bilinear map $b: E \times F \rightarrow G$ where $E, F, G$ are Banach spaces and $b$ is a bilinear operator satisfying continuity conditions. They establish a general version of the theorem for subseries convergent series and apply it to vector integration. There is a more general version given in [Sw7] where $E$ is a vector space, $F, G$ are locally convex spaces and $b$ is a bilinear map and multiplier convergent series are considered. Applications to multiplier convergent series in spaces of operators are given. Another generalization is given by Chen and $\mathrm{Li}([\mathrm{CL}])$ where $E, F$ are vector spaces, $G$ is a locally convex space and $b$ is what they call a bi-quasi-homogeneous operator. They consider multiplier convergent series of quasi-homogeneous operators. Li and Wang ([LW]) have considered the case when $E$ is a set and $F$ is a set of $G$ valued functions. They consider operator valued multiplier convergent series where the space of multipliers is vector valued. The case where $E, F$ are vector spaces, $G$ is a locally convex space and $b$ is a 
bilinear map is considered in [LS3]; general versions of the Orlicz-Pettis Theorem are established and numerous applications are given. A similiar treatment is given in Chapter 4 of [Sw6], pages 73-82. Li and Cho ([LC]) have used the general abstract setting above to obtain a generalization of an Orlicz-Pettis result of Kalton; we will consider this result later. Zheng, Cui and $\mathrm{Li}([\mathrm{ZCL}])$ have also considered abstract duality pairs in spaces with sectional operators and indicated applications to sequence spaces. By considering subseries convergent series only, we are able to treat the case of group valued series in our setup.

\section{Orlicz-Pettis Theorems.}

We now establish several versions of the Orlicz-Pettis Theorem for abstract triples. The classical version of the Orlicz-Pettis Theorem for normed spaces asserts that a series in a normed space which is subseries convergent in the weak topology of the space is subseries convergent in the norm topology ([Or], $[\mathrm{Pe}])$. The theorem has been extended to locally convex spaces and many other situations including topological groups. See [K1],[FL] for a discussion of the history of the subject. We refer to any result which asserts that a series which is subseries convergent in some weak topology is subseries convergent in a stronger topology as an Orlicz-Pettis Theorem.

Let $w(F, E)$ be the weakest topology on $F$ such that all of the maps $\{b(x, \cdot): x \in E\}$ from $F$ into $G$ are continuous. A subset $B \subset F$ is sequentially conditionally $w(F, E)$ compact if every sequence $\left\{y_{k}\right\} \subset B$ has a subsequence $\left\{y_{n_{k}}\right\}$ such that the sequence $\left\{x \cdot y_{n_{k}}\right\}=\left\{b\left(x, y_{n_{k}}\right)\right\}$ converges in $G$ for every $x \in E$ (this is terminology of Dinculeanu ([Di]).

The method of proof used in treating our versions of the Orlicz-Pettis Theorem relies on the Antosik-Mikusinski Matrix Theorem which we now state for convenience.

Theorem 9. (Antosik-Mikusinski) Let $G$ be an abelian topological group and $x_{i j} \in G$ for $i, j \in \mathbf{N}$. Suppose (I) $\lim _{i} x_{i j}=x_{j}$ exists for each $j$ and (II) for each increasing sequence of positive integers $\left\{m_{j}\right\}$ there is a subsequence $\left\{n_{j}\right\}$ of $\left\{m_{j}\right\}$ such that $\left\{\sum_{j=1}^{\infty} x_{i n_{j}}\right\}$ is Cauchy. Then $\lim _{i} x_{i j}=$ $x_{j}$ uniformly for $j \in \mathbf{N}$. In particular,

$$
\lim _{i} \lim _{j} x_{i j}=\lim _{j} \lim _{i} x_{i j}=0 \text { and } \lim _{i} x_{i i}=0 .
$$

A matrix $M=\left[x_{i j}\right]$ which satisfies conditions (I) and (II) of Theorem 9 is referred to as a $\mathcal{K}$ matrix.

For the proof, see [Sw5] 2.2, [Sw6]Appendix D. 
Theorem 10. If the series $\sum x_{j}$ is $w(E, F)$ subseries convergent, then the series $\sum_{j \in \sigma} x_{j} \cdot y$ converge uniformly for $y \in B$ and $\sigma \subset \mathbf{N}$, where $B$ is any sequentially conditionally $w(F, E)$ compact subset $B \subset F$ [ that is, for every closed neighborhood of $0, U$, in $G$ there exists $N$ such that $\sum_{j \in \sigma} x_{j} \cdot y \in U$ whenever $y \in B$ and $\min \sigma>N$; a strong form of unordered convergence for the series].

Proof. If the conclusion fails to hold, there exists a closed neighborhood $U$ of 0 such that for every $k$ there exist $\sigma_{k}$ with $\min \sigma_{k}>k$ and $y_{k} \in B$ such that $\sum_{j \in \sigma_{k}} x_{j} \cdot y_{k} \notin U$. Put $k_{1}=1$ so we have $\sum_{j \in \sigma_{1}} x_{j} \cdot y_{1} \notin U$. We may assume that $\sigma_{1}$ is finite since $U$ is closed. Put $k_{2}=\max \sigma_{1}$. Apply the condition above to $k_{2}$ to obtain $\sum_{j \in \sigma_{2}} x_{j} \cdot y_{2} \notin U$ with $\sigma_{2}$ finite, $\min \sigma_{2}>k_{2}$ and $y_{2} \in B$. This construction produces finite sequences $\left\{\sigma_{k}\right\}$ with $\min \sigma_{k+1}>\max \sigma_{k}$ and $\left\{y_{k}\right\} \subset B$ satisfying

$$
\text { (\&) } \sum_{j \in \sigma_{k}} x_{j} \cdot y_{k} \notin U
$$

There exists a subsequence $\left\{y_{n_{k}}\right\}$ such that $\lim x \cdot y_{n_{k}}$ exists for every $x \in E$. Consider the matrix

$$
M=\left[m_{i j}\right]=\left[\sum_{l \in \sigma_{j}} x_{l} \cdot y_{n_{i}}\right] .
$$

We claim that $M$ is a $\mathcal{K}$ matrix. The columns of $M$ converge and for every subsequence $\left\{r_{j}\right\}$ the subseries $\sum_{j=1}^{\infty} \sum_{l \in \sigma_{r_{j}}} x_{l}$ is $w(E, F)$ convergent and

$$
\lim _{i} \sum_{j=1}^{\infty} m_{i r_{j}}=\lim _{i} \sum_{j=1}^{\infty} \sum_{l \in \sigma_{r_{j}}} x_{l} \cdot y_{n_{i}}
$$

exists. Therefore, $M$ is a $\mathcal{K}$ matrix whose diagonal converges to 0 by the Antosik-Mikusinski Theorem. But, this contradicts (\&).

A subset $B \subset F$ is sequentially relatively $w(F, E)$ compact if every sequence $\left\{y_{k}\right\} \subset B$ has a subsequence $\left\{y_{n_{k}}\right\}$ and there exists $y \in F$ such that $\lim _{k} x \cdot y_{n_{k}}=x \cdot y$ for every $x \in E$. A sequentially relatively $w(F, E)$ compact is obviously sequentially conditionally $w(F, E)$ compact so the result above holds for this family of subsets of $F$.

The unordered convergence form of the conclusion of Theorem 10 is useful in treating the Hahn-Schur Theorem given in Case 18.

We consider the theorem for $w(F, E)$ compact subsets. 
Theorem 11. Let $G$ be metrizable under the metric $\rho$. If $\sum x_{j}$ is $w(E, F)$ subseries convergent, then the series $\sum_{j \in \sigma} x_{j} \cdot y$ converge uniformly for $y \in B$ and $\sigma \subset \mathbf{N}$, where $B$ is any $w(F, E)$ compact subset $B \subset F$.

Proof. Let $B$ be $w(F, E)$ compact. Define an equivalence relation on $B$ by $y \sim z$ iff $x_{j} \cdot y=x_{j} \cdot z$ for every $j$. Let $\widehat{B}$ be the collection of equivalence classes and $\widehat{y}$ the equivalence class to which $y$ belongs. Define a metric $d$ on $\widehat{B}$ by

$$
d(\widehat{y}, \widehat{z})=\sum_{j=1}^{\infty} \frac{1}{2^{j}} \frac{\rho\left(x_{j} \cdot y, x_{j} \cdot z\right)}{1+\rho\left(x_{j} \cdot y, x_{j} \cdot z\right)}
$$

so $\widehat{y_{\alpha}} \rightarrow \widehat{y}$ with respect to $d$ iff $\lim _{\alpha} x_{j} \cdot y_{\alpha}=x_{j} \cdot y$ for all $j$.

Let $\mathcal{S}$ be the set of partial sums of the series $\sum x_{j}$; i.e., $\mathcal{S}=\left\{\sum_{j \in \sigma} x_{j}\right.$ : $\sigma \subset \mathbf{N}\}$. Note that if $y, z \in B$ and $y \sim z$, then $x_{\sigma} \cdot y=x_{\sigma} \cdot z$ for all $\sigma \subset \mathbf{N}$. Thus, $(\mathcal{S}, \widehat{B} ; G)$ is an abstract triple under the map $\left(x_{\sigma}, \widehat{y}\right)=x_{\sigma} \cdot y$. Since $B$ is $w(F, E)$ compact, $\widehat{B}$ is $w(\widehat{B}, \mathcal{S})$ compact. [If $\left\{\widehat{y_{\alpha}}\right\}$ is a net in $\widehat{B}$, then $\left\{y_{\alpha}\right\}$ is a net in $B$ and so has a subnet $\left\{y_{\beta}\right\}$ which is $w(F, E)$ convergent to some $y \in B$ and then $x \cdot y_{\beta} \rightarrow x \cdot y$ for $x \in E$. In particular, $x_{\sigma} \cdot y_{\beta} \rightarrow x_{\sigma} \cdot y$ for every $\sigma$ so $\left\{\widehat{y_{\beta}}\right\}$ is $w(\widehat{B}, \mathcal{S})$ convergent to $\widehat{y}$.] The inclusion $(\widehat{B}, w(\widehat{B}, \mathcal{S})) \rightarrow(\widehat{B}, d)$ is continuous so $d=w(\widehat{B}, \mathcal{S})$ on $\widehat{B}$. Now $\sum x_{j}$ is $w(\mathcal{S}, \widehat{B})$ subseries convergent and $\widehat{B}$ is $w(\widehat{B}, \mathcal{S})$ sequentially compact since this topology is metrizable so it follows from the previous Orlicz-Pettis Theorem that the series $\sum x_{j}$ converges uniformly on $\widehat{B}$ and, therefore, on $B$.

We will discuss applications of Theorems 10 and 11 to locally convex spaces in Case 16 below.

A result of Kalton ([K2]) asserts that if $\tau$ is a separable polar topology on $E$ from the dual pair $E, F$, then any series $\sum x_{j}$ in $E$ which is $\sigma(E, F)$ subseries convergent is $\tau$ subseries convergent. If $\tau$ is the polar topology of uniform convergence on the family $\mathcal{A}$ of $\sigma(F, E)$ bounded subsets of $F$, then for every $x \in E$ and $A \in \mathcal{A}$ the set $\{x \cdot y: y \in A\}$ is sequentially relatively compact in the scalar field. We give an abstraction of this condition and use it to give a generalization of Kalton's result to abstract triples.

Definition 12. $A$ subset $B \subset F$ is conditionally sequentially compact at each $x \in E$ if $\{x \cdot y: y \in B\}=x \cdot B$ is conditionally sequentially compact in $G$ for every $x \in E$.

Note that if $B \subset F$ is sequentially conditionally $w(F, E)$ compact, then $B$ is sequentially conditionally compact at each $x \in E$. Under a separability assumption, the converse holds. 
Theorem 13. Let $G$ be sequentially complete. Let $\mathcal{F}$ be a family of subsets of $F$ such that each member of $\mathcal{F}$ is sequentially conditionally compact at each $x \in E$ and let $\tau$ be the topology on $E$ of uniform convergence on the members of $\mathcal{F}$. If $(E, \tau)$ is separable, then each member of $\mathcal{F}$ is sequentially conditionally $w(F, E)$ compact.

Proof. Let $D=\left\{d_{k}: k \in \mathbf{N}\right\}$ be $\tau$ dense in $E$. Let $B \in \mathcal{F}$ and $\left\{y_{k}\right\} \subset B$. Since $B$ is sequentially conditionally compact at each $x \in E$, by the diagonalization procedure $\left\{y_{k}\right\}$ has a subsequence $\left\{y_{n_{k}}\right\}$ such that the sequence $\left\{d \cdot y_{n_{k}}\right\}$ converges in $G$ for every $d \in D$. Let $x \in E$. There is a net $\left\{d^{\alpha}\right\} \subset D$ which is $\tau$ convergent to $x$ so $\lim d^{\alpha} \cdot y=x \cdot y$ uniformly for $y \in B$. Let $U$ be a neighborhood of 0 in $G$ and pick a symmetric neighborhood $V$ of 0 in $G$ such that $V+V+V \subset U$. There exists $\beta$ such that $d^{\beta} \cdot y_{n_{k}}-x \cdot y_{n_{k}} \in V$ for all $k$. Since $\left\{d^{\beta} \cdot y_{n_{k}}\right\}_{k}$ converges, there exists $N$ such that $k, j \geq N$ implies $d^{\beta} \cdot y_{n_{k}}-d^{\beta} \cdot y_{n_{j}} \in V$. Hence, if $k, j \geq N$, then

$x \cdot y_{n_{k}}-x \cdot y_{n_{j}}=x \cdot y_{n_{k}}-d^{\beta} \cdot y_{n_{k}}+d^{\beta} \cdot y_{n_{k}}-d^{\beta} \cdot y_{n_{j}}+d^{\beta} \cdot y_{n_{j}}-x \cdot y_{n_{j}} \in V+V+V \subset U$

so $\left\{x \cdot y_{n_{k}}\right\}$ is Cauchy and, therefore, convergent since $G$ is sequentially complete. Hence, $B$ is sequentially conditionally $w(F, E)$ compact.

Corollary 14. If the conditions of Theorem 13 hold and $\sum x_{j}$ is $w(E, F)$ subseries convergent, then $\sum x_{j}$ is $\tau$ subseries convergent.

Proof. The result is immediate from Theorems 13 and 10.

The separability assumption in Theorem 13 is important.

Example 15. The series $\sum e^{j}$ is $\sigma\left(l^{\infty}, l^{1}\right)$ subseries convergent but is not $\beta\left(l^{\infty}, l^{1}\right)=\|\cdot\|_{\infty}$ subseries convergent.

\section{Applications}

We begin with the Orlicz-Pettis Theorem for locally convex spaces.

Case 16. Let $E$ be a Hausdorff locally convex space with dual $E^{\prime}$. Suppose $\sum x_{j}$ is subseries convergent with respect to $\sigma\left(E, E^{\prime}\right)$. We may assume that $E$ is separable by replacing $E$ with the span of $\left\{x_{j}\right\}$, if necessary. Let $\gamma\left(E, E^{\prime}\right)\left(\lambda\left(E, E^{\prime}\right) ; \tau\left(E, E^{\prime}\right)\right)$ be the topology of uniform convergence on the 
sequentially conditionally $\sigma\left(E^{\prime}, E\right)$ compact subsets of $E^{\prime}\left(\sigma\left(E^{\prime}, E\right)\right.$ compact subsets; convex $\sigma\left(E^{\prime}, E\right)$ compact subsets). It follows from Theorem 10 that $\sum x_{j}$ is $\gamma\left(E, E^{\prime}\right)$ subseries convergent. Also, when $E$ is separable the $\sigma\left(E^{\prime}, E\right)$ topology on any $\sigma\left(E^{\prime}, E\right)$ compact subset is metrizable ([Wi]9.5.3) so any $\sigma\left(E^{\prime}, E\right)$ compact subset is sequentially $\sigma\left(E^{\prime}, E\right)$ compact and it follows from Theorem 10 that $\sum x_{j}$ is subseries convergent with respect to $\lambda\left(E, E^{\prime}\right)$ and, therefore, subseries convergent with respect to $\tau\left(E, E^{\prime}\right)$, the Mackey topology.

The locally convex version of the Orlicz-Pettis Theorem for the Mackey topology is due to McArthur ([Mc]); the version for the topology $\lambda\left(E, E^{\prime}\right)$ is due to Bennett and Kalton $([\mathrm{BK}])$ and the version for $\gamma\left(E, E^{\prime}\right)$ is due to Dierolf ([Die]). Kalton's version of the Orlicz-Pettis Theorem will be considered in Case 25.

Next, we consider a theorem of Nikodym for group valued measures.

Case 17. (Nikodym Convergence Theorem) Let $\Sigma$ be a $\sigma$ algebra of subsets of a set $S$ and let $\mu_{n}: \Sigma \rightarrow G$ be a countably additive measure for $n \in \mathbf{N}$ and set $\mathcal{M}=\left\{\mu_{n}\right\}$. Suppose $\lim _{n} \mu_{n}(A)=\mu(A)$ exists for every $A \in \Sigma$. The Nikodym Theorem asserts that (i) $\mu$ is countable additive and (ii) $\left\{\mu_{n}\right\}$ is uniformly countably additive. We observe that (ii) follows from Example 3 and Theorem 10. Let $\left\{A_{j}\right\}$ be a pairwise disjoint sequence from $\Sigma$. Then the series $\sum A_{j}$ is $w(\Sigma, \mathcal{M})$ subseries convergent by the countable additivity of the $\mu_{n}$. Also, the sequence $\left\{\mu_{n}\right\}$ is sequentially conditionally $w\left(\Sigma,\left\{\mu_{n}\right\}\right)$ compact by hypothesis. Hence, from Theorem 10 it follows that the series $\sum_{j=1}^{\infty} \mu_{n}\left(A_{j}\right)$ converge uniformly for $n \in \mathbf{N}$. But, this is just (ii). Condition (i) follows from (ii).

For the case of the Nikodym Theorem for groups see [AS1]. Using a result of Drewnowski, the Nikodym Convergence Theorem can be extened to strongly additive set functions. An additive set function $\mu: \Sigma \rightarrow G$ is strongly additive if $\mu\left(A_{j}\right) \rightarrow 0$ for every disjoint sequence $\left\{A_{j}\right\} \subset \Sigma$; see [Sw5] 2.3.5 for details.

We note in passing that a version of the Nikodym Boundedness Theorem can be obtained from the Nikodym Convergence Theorem. Suppose $\mathcal{M}$ is a family of countably additive scalar valued set functions defined on $\Sigma$ which is pointwise bounded on $\Sigma$. The Nikodym Boundedness Theorem asserts that $\mathcal{M}$ is uniformly bounded on $\Sigma$ ([DU]). To show that $\mathcal{M}$ is uniformly bounded on $\Sigma$ it suffices to show that $\left\{\mu_{n}\left(A_{n}\right)\right\}$ is bounded for every $\left\{\mu_{n}\right\} \subset$ $\mathcal{M}$ and every pairwise disjoint sequence $\left\{A_{n}\right\} \subset \Sigma$ (see $[\mathrm{Sw} 5] 4.7 .1$; we 
develop a more general result later in Theorem 34). $\left\{\mu_{n}\left(A_{n}\right)\right\}$ is bounded if $\frac{1}{n} \mu_{n}\left(A_{n}\right) \rightarrow 0$. Since $\mathcal{M}$ is pointwise bounded, $\frac{1}{n} \mu_{n}(A) \rightarrow 0$ for every $A \in \Sigma$ so the Nikodym Convergence Theorem implies the series $\sum_{j=1}^{\infty} \frac{1}{n} \mu_{n}\left(A_{j}\right)$ converge uniformly for $n \in \mathbf{N}$. In particular, $\frac{1}{n} \mu_{n}\left(A_{n}\right) \rightarrow 0$ as desired. The version of the theorem for locally convex valued measures follows from the scalar version and the Uniform Boundedness Theorem. We consider a more general version of the theorem in Corollary 36 .

We next consider a version of the Hahn-Schur Theorem for group valued series (see [AS1]; [Sw6]8.1).

Case 18. (Hahn-Schur Theorem) Let $\sum_{j} x_{i j}$ be a subseries convergent series in $G$ for every $i \in \mathbf{N}$ and suppose $\lim _{i} \sum_{j \in \sigma} x_{i j}$ exists for every $\sigma \subset \mathbf{N}$. Set $x_{j}=\lim _{i} x_{i j}$. The subseries version of the Hahn-Schur Theorem then assets that (i) $\sum x_{j}$ is subseries convergent, (ii) the series $\sum_{j \in \sigma} x_{i j}$ converge uniformly for $i \in \mathbf{N}, \sigma \subset \mathbf{N}$, and (iii) $\lim _{i} \sum_{j \in \sigma} x_{i j}=\sum_{j \in \sigma} x_{j}$ uniformly for $\sigma \subset \mathbf{N}$. We show that (ii) follows directly from Theorem 10. Let $E$ be the power set of $\mathbf{N}$, define $f_{i}: E \rightarrow G$ by $f_{i}(\sigma)=\sum_{j \in \sigma} x_{i j}$ and set $F=\left\{f_{i}: i \in \mathbf{N}\right\}$. Then $(E, F: G)$ is an abstract triple and the (formal) series $\sum_{j} j$ is $w(E, F)$ subseries convergent with $\sum_{j} f_{i}(j)=\sum_{j} x_{i j}$. By hypothesis, $F$ is sequentially conditionally $w(F, E)$ compact so from Theorem 10 , it follows that (ii) holds. Conditions (i) and (iii) follow immediately from (ii).

The usual scalar version of the theorem can be obtained easily from Case 18 (see [AS1]8.2; [Sw5]8.1).

We now consider subseries convergence in the space of continuous linear operators.

Case 19. Let $E, G$ be topological vector spaces with $G$ metrizable and consider the abstract triple $(L(E, G), E: G)$ as in Example 6. Suppose that $\sum T_{j}$ is a series in $L(E, G)$ which is subseries convergent in the strong operator topology. Note that any subset $B \subset E$ which is compact in $E$ is $w(E, L(E, G))$ compact so the series $\sum T_{j}$ is subseries convergent in $L_{c}(E, G)$, the topology of uniform convergence on compact subsets of $E$, by Theorem 11 .

We consider subseries convergence with respect to pointwise convergence in spaces of continuous functions.

Case 20. Let $\Omega$ be a sequentially compact topological space and let $S C(\Omega, G)$ be the space of all sequentially continuous functions from $\Omega$ into $G$. Suppose 
$\sum f_{j}$ is a series in $S C(\Omega, G)$ which is subseries convergent in the topology of pointwise convergence on $G$. We claim the series $\sum f_{j}(t)$ is subseries convergent uniformly for $t \in \Omega$. To see this consider the abstract triple $(S C(\Omega, G), \Omega: G)$ under the map $(f, t)=f(t)$. The series $\sum f_{j}$ is subseries convergent with respect to $w(S C(\Omega, G), \Omega)$ and the set $\Omega$ is $w(\Omega, S C(\Omega, G))$ sequentially compact since $\Omega$ is sequentially compact so the claim follows from Theorem 10.

Case 21. Let $\Omega$ be a topological space, $G$ be metrizable and $C(\Omega, G)$ the space of continuous functions from $\Omega$ to $G$. Suppose $\sum f_{j}$ is a series in $C(\Omega, G)$ which is subseries convergent in the topology of pointwise convergence on $\Omega$. We claim that the series is subseries convergent in the topology of uniform convergence on compact subsets of $\Omega$. To see this consider the abstract triple $(C(\Omega, G), \Omega: G)$ under the map $(f, t) \rightarrow f(t)$. The series $\sum f_{j}$ is $w(C(\Omega, G), \Omega)$ subseries convergent and any compact subset of $\Omega$ is $w(\Omega, C(\Omega, G))$ compact so the claim follows from Theorem 11.

Theorems of this type relative to pointwise convergent series in spaces of continuous functions were established in [Th] and [Sw2].

Case 22. Let $X$ be a topological vector space and assume that $E$ is a vector space of $X$ valued sequences which contains the space $c_{00}(X)$ of all $X$ valued sequences which are eventually 0 . If $z \in X$ and $k \in \mathbf{N}$, let $e^{k} \otimes z$ be the sequence with $z$ in the $k^{\text {th }}$ coordinate and 0 in the other coordinates. Assume that $E$ has a vector topology under which the coordinate mappings $\left\{x_{k}\right\} \rightarrow e^{k} \otimes x_{k}$ form $E$ into $E$ are continuous (i.e., $E$ is a $K$-space) and for every $x=\left\{x_{k}\right\} \in E$ we have $x=\sum_{k=1}^{\infty} e^{k} \otimes x_{k}$ (i.e., $E$ is an $A K-$ space). Suppose that $\sum x^{j}$ is a series in $E$ which is subseries convergent with respect to the topology of coordinatewise convergence. We claim that the series $\sum x^{j}$ is subseries convergent in the original topology of $E$. To see this consider the following abstract triple. Define $P_{k}: E \rightarrow E$ by $P_{k} x=\sum_{i=1}^{k} e^{i} \otimes x_{i}$ so $P_{k} x \rightarrow x$ in $E$ for every $x \in E$ by the AK assumption. Set $F=\left\{P_{k}: k \in \mathbf{N}\right\}$ and note that $(E, F: E)$ is an abstract triple under the map $\left(x, P_{k}\right) \rightarrow P_{k} x$ and that the series $\sum x^{j}$ is subseries convergent with respect to $w(E, F)$. The set $F$ is sequentially conditionally $w(F, E)$ compact by the AK hypothesis so it follows from Theorem 10 that the series $\sum_{j=1}^{\infty} P_{k} x^{j}$ is subseries convergent uniformly for $k \in \mathbf{N}$. To establish the claim let $U$ be a closed neighborhood of o in $E$. Set $s^{n}=\sum_{j=1}^{n} x^{j}$ and $s=\sum_{j=1}^{\infty} x^{j}$, where this is the coordinate sum of the series. Since $\lim _{n} P_{k} s^{n}=P_{k} s$ uniformly for $k \in \mathbf{N}$, there exists $N$ such that $P_{k} s^{n}-P_{k} s \in$ 
$U$ for $n \geq N, k \in \mathbf{N}$. Fixing $n$ and letting $k \rightarrow \infty$ gives $s^{n}-s \in U$ for $n \geq N$. Since the same argument can be applied to any subsequence, the claim follows. This result applies to such sequence spaces as $l^{p}(X)$ , $1<p<\infty$, and $c_{0}(X)$.

We next consider a result of Stiles for spaces with a Schauder basis.

Case 23. (Stiles) Let $E$ be a topological vector space with a Schauder basis $\left\{b_{k}\right\}$ and coordinate functionals $\left\{f_{k}\right\}$. For each $k$ let $P_{k}: E \rightarrow E$ be the projection $P_{k} x=\sum_{j=1}^{k}\left\langle f_{j}, x\right\rangle b_{j}$. If $\sum x_{j}$ is subseries convergent with respect to $\sigma\left(E,\left\{f_{j}\right\}\right)$, we claim that the series is subseries convergent in the original topology of $E$. To see this, set $F=\left\{P_{j}: j \in \mathbf{N}\right\}$ and consider the abstract triple $(E, F: E)$ under the map $\left(x, P_{j}\right) \rightarrow P_{j} x$. Then $\sum x_{j}$ is $w(E, F)$ subseries convergent and $F$ is sequentially conditionally $w(F, E)$ compact since $P_{k} x \rightarrow x$ so the series $\sum_{j=1}^{\infty} P_{k} x_{j}$ is subseries convergent uniformly for $k \in \mathbf{N}$ by Theorem 10. As in Case 22 this establishes the claim.

Stiles established this result for metrizable, complete spaces ([St]; see also [B],[Sw4]); the metrizable and completeness assumptions were later removed ([Sw5]10.4.1).

The result in Case 23 can be generalized somewhat. Assume that $E$ is a topological vector space and there exist a sequence of linear operators $\left\{P_{k}\right\}$ such that for each $x \in E$ we have $x=\sum_{k=1}^{\infty} P_{k} x$ with convergence in $E$. When the $\left\{P_{k}\right\}$ are continuous, then $\left\{P_{k}\right\}$ is called a Schauder decomposition ([LT]). Then the proof in Case 22 shows that if a series is subseries convergent in $w\left(E,\left\{P_{k}\right\}\right)$, then the series is subseries convergent in the topology of $E$.

We next consider a result of Tweddle.

Case 24. (Tweddle) Let $E, F$ be a pair of vector spaces in duality. Let $\mathcal{E}$ be the family of all $\sigma(E, F)$ subseries convergent series in $E$ and let $E^{\#}$ be all linear functionals $x^{\prime}$ on $E$ such that $\sum_{j=1}^{\infty}\left\langle x^{\prime}, x_{j}\right\rangle=\left\langle x^{\prime}, \sum_{j=1}^{\infty} x_{j}\right\rangle$ for all $\left\{x_{j}\right\} \in \mathcal{E}$, where $\sum_{j=1}^{\infty} x_{j}$ is the $\sigma(E, F)$ sum of the series. Then $E, E^{\#}$ form a dual pair and each $\left\{x_{j}\right\} \in \mathcal{E}$ is $\sigma\left(E, E^{\#}\right)$ subseries convergent. It follows from Case 16 that every $\left\{x_{j}\right\} \in \mathcal{E}$ is subseries convergent in the Mackey topology $\tau\left(E, E^{\#}\right)$ of uniform convergence on convex $\sigma\left(E, E^{\#}\right)$ compact subsets of $E^{\#}$. This is the Tweddle topology of $E$ and Tweddle has shown that this is the strongest locally convex topology on $E$ which has the same $\sigma(E, F)$ subseries convergent series ([Tw]). 
The topology of Tweddle can also be extended to our abstract setting. Let $(E, F: G)$ be an abstract triple and let $\mathcal{E}$ be the family of all $w(E, F)$ subseries convergent series. Let $E^{\#}$ be all functions $f: E \rightarrow G$ such that $f\left(\sum_{j=1}^{\infty} x_{j}\right)=\sum_{j=1}^{\infty} f\left(x_{j}\right)$ for every $\left\{x_{j}\right\} \in \mathcal{E}$. Then $\left(E, E^{\#}: G\right)$ form an abstract triple and each $\left\{x_{j}\right\} \in \mathcal{E}$ is $w\left(E, E^{\#}\right)$ subseries convergent. If $G$ is metrizable, it follows from Theorem 11 that each $\left\{x_{j}\right\} \in \mathcal{E}$ is subseries convergent in the topology of uniform convergence on $w\left(E^{\#}, E\right)$ compact subsets of $E^{\#}$.

We indicate applications of Theorem 13. First, we consider a result of Kalton $([\mathrm{K} 2])$.

Case 25. (Kalton) Let $E, F$ be a dual pair of vector spaces and $\tau$ a polar topology on $E$ from this duality which is separable. If $\tau$ is the polar topology of uniform convergence on the family $\mathcal{A}$ of $\sigma(F, E)$ bounded subsets of $F$, then every subset $A$ of $\mathcal{A}$ is sequentially conditionally compact at each $x \in$ $E$ so by Theorem 13 any series in $E$ which is $\sigma(E, F)$ subseries convergent is $\tau$ subseries convergent.

Next, we consider the space of compact operators.

Case 26. Let $E, G$ be Hausdorff topological vector spaces and $K(E, G)$ the space of all continuous linear operators which carry bounded subsets of $E$ into sequentially conditionally compact subsets of $G$. If $E, G$ are Banach spaces $K(E, G)$ is the space of compact operators. Then $(K(E, G), E: G)$ is an abstract triple and if $\mathcal{B}$ is the family of bounded subsets of $E$ each $B \in \mathcal{B}$ is sequentially conditionally compact at each $T \in K(E, G)$. The topology $w(K(E, G), E)$ is just the strong operator topology. Let $K_{b}(E, G)$ be the topology of uniform convergence on the members of $\mathcal{B}$; if $E, G$ are normed spaces this is just the uniform operator topology of $K(E, G)$. From Theorem 13 it follows that if $\mathcal{F}(E, G)$ is any separable subspace of $K_{b}(E, G)$, then any series in $\mathcal{F}(E, G)$ which is subseries convergent in the strong operator topology is subseries convergent in $K_{b}(E, G)$.

We indicate situations where Case 26 is applicable. Let $E, G$ be Banach spaces and let $\mathcal{F}(E, G)$ be the space of operators with finite dimensional range. Thus, every operator $T \in \mathcal{F}(E, G)$ has a representation $T x=$ $\sum_{j=1}^{n}\left\langle x_{j}^{\prime}, x\right\rangle y_{j}$ with $x_{j}^{\prime} \in E^{\prime}, y_{j} \in G$. If $E^{\prime}$ and $G$ are separable, then $\mathcal{F}(E, G)$ is a separable subspace of $K_{b}(E, G)$ so Case 26 applies. If, in addition, either $E^{\prime}$ or $G$ has the approximation property, then $\mathcal{F}(E, G)$ is 
dense in $K_{b}(E, G)$ ([LT]1.e.4 or 1.e.5) so Case 26 also applies in this case to $K_{b}(E, G)$.

Another situation where Case 26 applies is given as follows. Let $E$ be a metrizable nuclear space and $G$ be separable. Then any continuous linear operator from $E$ into $G$ is in $K_{b}(E, G)$ since bounded subsets of $E$ are relatively compact. Now $E$ is separable ([G]II.VI.5) and $E_{b}^{\prime}$ is separable ([G]II.VI.12) so $L_{b}(E, G)$ is separable ([G]III.II.11.b and 13.c) and Case 26 applies.

Also, if $E$ is dual nuclear (i.e., the strong dual of $E$ is nuclear) and $G$ is nuclear, then $L_{b}(E, G)$ is nuclear $([\mathrm{Pi}] 5.5 .1)$ and, therefore, separable so Case 26 applies.

Finally, If $E, G$ are normed spaces and $E^{\prime}, G$ are separable, then $\mathcal{F}(E, G)$ is separable under the nuclear norm $\nu$ on $\mathcal{F}(E, G)$ (see $[\mathrm{Pi}] 3.1$ ) so the space of nuclear operators $\mathcal{N}(E, G)$ is separable under the nuclear norm $\nu$ ([Pi]3.1.4) and Case 26 applies. Similar remarks apply to the space of Hilbert-Schmidt (absolutely summing) operators on Hilbert spaces ([Pi] 2.5).

Some of the results of this case were announced without proofs in [LC].

We consider another result related to compact operators and a family of operators introduced by A. Mohsen. Let $X, Y, Z$ be normed spaces and let $W^{*}\left(Y^{\prime}, Z\right)$ be the space of all sequentially weak*-\|.\| continuous linear operators from $Y^{\prime}$ into $Z$ (these operators were introduced and studied by Mohsen ([Mo] $)$ and were shown to be bounded).

Case 27. Let $B(Z)$ denote the unit ball of any normed space $Z$. Assume $B\left(Y^{\prime}\right)$ is weak* sequentially compact and that the series $\sum U_{j}$ is subseries convergent in the strong operator topology of $W^{*}\left(Y^{\prime}, Z\right)$. Consider the abstract triple $\left(W^{*}\left(Y^{\prime}, Z\right), B\left(Y^{\prime}\right): Z\right)$ under the map $\left(U, y^{\prime}\right) \rightarrow U y^{\prime}$. Then the series $\sum U_{j}$ is $w\left(W^{*}\left(Y^{\prime}, Z\right), B\left(Y^{\prime}\right)\right)$ subseries convergent and $B\left(Y^{\prime}\right)$ is $w\left(B\left(Y^{\prime}\right), W^{*}\left(Y^{\prime}, Z\right)\right)$ sequentially compact since if $\left\{y_{j}^{\prime}\right\} \subset B\left(Y^{\prime}\right)$, then there is a subsequence $\left\{y_{n_{j}}^{\prime}\right\}$ which is weak* convergent to some $y^{\prime} \in B\left(Y^{\prime}\right)$ and $\left\|U y_{n_{j}}^{\prime}-U y^{\prime}\right\| \rightarrow 0$ by the definition of $W^{*}\left(Y^{\prime}, Z\right)$. Hence, by Theorem 10 the series $\sum_{j=1}^{\infty} U_{j} y^{\prime}$ converge uniformly for $y^{\prime} \in B\left(Y^{\prime}\right)$ and similarly for any subseries. That is, the series $\sum U_{j}$ is subseries convergent in norm.

As a special case of Case 27, we can obtain a result of Kalton ([K3]). We say a normed space $Z$ has the Diestel-Faires property (DF property) if any weak* subseries convergent series $\sum z_{j}$ in $Z^{\prime}$ is norm subseries convergent ([DF]; Diestel and Faires have characterized the Banach spaces with DF as the spaces $Z$ whose dual does not contain a copy of $l^{\infty}$ ). 
Case 28. (Kalton) Let $\sum T_{j}$ be subseries convergent in the weak operator topology of $K(X, Y)$ and assume $X$ has the DF property. Since each $T_{j}$ has separable range we may assume that $Y$ is separable by replacing $Y$ with the union of the ranges of the $T_{j}$, if necessary. For each $z^{\prime} \in Y^{\prime}$ the series $\sum T_{j}^{\prime} z^{\prime}$ is weak ${ }^{*}$ subseries convergent in $X^{\prime}$ and by the DF property is norm subseries convergent. Let $K^{\prime}(X, Y)$ be $\left\{T^{\prime} \in K\left(Y^{\prime}, X^{\prime}\right): T \in K(X, Y)\right\}$ and consider the abstract triple $\left(K^{\prime}(X, Y), B\left(Y^{\prime}\right) ; X^{\prime}\right)$. The series $\sum T_{j}^{\prime}$ is $w\left(K^{\prime}(X, Y), B\left(Y^{\prime}\right)\right)$ subseries convergent by the observation above and the ball of $Y^{\prime}$ is weak ${ }^{*}$ sequentially compact since $Y$ is separable. Also, $K^{\prime}(X, Y) \subset W^{*}\left(Y^{\prime}, X^{\prime}\right)$ ([DS]VI.5.6) so Case 27 implies that the series $\sum T_{j}^{\prime}$ is norm subseries convergent and, hence, the series $\sum T_{j}$ is norm subseries convergent.

We show that a version of the Uniform Boundedness Principle can be obtained from the Orlicz-Pettis Theorem.

Case 29. (Uniform Boundedness Principle) Let $G$ be a locally convex space and let $E$ be a sequentially complete locally convex space. Let $\Gamma$ be a subset of $L(E, G)$ which is pointwise bounded on $E$. We claim that $\Gamma$ is uniformly bounded on bounded subsets of $E$; a version of the Uniform Boundedness Principle (see [Sw5] 4.2 for this version; if $E$ is metrizable, $\Gamma$ is equicontinuous which is a more familiar conclusion of the Uniform Boundedness Principle). Suppose there exists a bounded subset $B$ of $E$ such that $\Gamma(B)$ is not bounded. Then there exists a continuous seminorm $p$ on $G$ such that

$$
\sup \{p(T x): T \in \Gamma, x \in B\}=\infty .
$$

Pick $T_{k} \in \Gamma$ and $x_{k} \in B$ such that

$$
\text { (*) } p\left(T_{k} x_{k}\right)>2^{2 k} \text {. }
$$

Since $\left\{x_{k}\right\}$ is bounded, the series $\sum_{k} x_{k} / 2^{k}$ is absolutely convergent and, therefore, subseries convergent in $E$ by the sequential completeness hypothesis. Set $F=\left\{T_{k} / 2^{k}: k \in \mathbf{N}\right\}$ and consider the abstract triple $(E, F: G)$ under the map $(x, T) \rightarrow T x$. Since the series $\sum_{k} x_{k} / 2^{k}$ is subseries convergent in $E$, the series is $w(E, F)$ subseries convergent. For each $x \in E$ the sequence $\left\{T_{k} x\right\}$ is bounded by hypothesis so $T_{k} x / 2^{k} \rightarrow 0$ which implies that that the sequence $\left\{T_{k} / 2^{k}\right\}$ is sequentially relativelyw $(F, E)$ compact; i.e., $F$ is sequentially relatively $w(F, E)$ compact. The Orlicz-Pettis Theorem 10 implies that the series $\sum_{j=1}^{\infty}\left(T_{k} / 2^{k}\right)\left(x_{j} / 2^{j}\right)$ converge uniformly for $k \in \mathbf{N}$. In particular, $T_{k} x_{k} / 2^{2 k} \rightarrow 0$ in $G$. But, this contradicts (*). 
The proof of Case 29 also gives an operator version of the BanachMackey Theorem ([Wi]10.4.8; [Sw5]4.2.7).

Case 30. (Banach-Mackey) Namely, if $\Gamma \subset L(E, G)$ is pointwise bounded on $E$ and $B \subset E$ is bounded, absolutely convex and sequentially complete, then $\Gamma(B)$ is bounded. Thus, if $E$ is a sequentially complete locally convex space, then weakly bounded subsets of $E^{\prime}$ are strongly bounded; this is the usual version of the Banach-Mackey Theorem.

A theorem of Mazur and Orlicz asserts that a separately continuous bilinear mapping from the product of two metric linear spaces one of which is complete is (jointly) continuous ([MO]). We show that the Orlicz-Pettis Theorem can be used to derive a similiar result.

Case 31. Let $E, G$ be locally convex spaces and $F$ be a topological vector space. Assume that $E$ is sequentially complete. Let $b: E \times F \rightarrow G$ be a bilinear separately continuous map. If $A \subset E, B \subset F$ are bounded, then $b(A, B)$ is bounded (i.e., $b$ is a bounded bilinear map). If the conclusion fails to hold, there is a continuous seminorm $p$ on $G$ such that

$$
\sup \{p(x, y): x \in A, y \in B\}=\infty .
$$

Pick $x_{k} \in A, y_{k} \in B$ such that

$$
\text { (\#) } p\left(x_{k}, y_{k}\right)>2^{2 k}
$$

and consider the abstract triple $(E, F: G)$ under the map $b$. The series $\sum x_{k} / 2^{k}$ is absolutely convergent in $E$ since $A$ is bounded and, therefore, the series is subseries convergent by the sequential completeness assumption. Since $b(\cdot, y)$ is continuous, the series $\sum x_{k} / 2^{k}$ is $w(E, F)$ subseries convergent. Also, $y_{k} / 2^{k} \rightarrow 0$ in $F$ since $B$ is bounded and $y_{k} / 2^{k} \rightarrow 0$ in $w(F, E)$ since $b(x, \cdot)$ is continuous. Therefore, $\left\{y_{k} / 2^{k}\right\}$ is $w(F, E)$ relatively compact. By Theorem 10 the series $\sum_{j=1}^{\infty} b\left(x_{j} / 2^{j}, y_{k} / 2^{k}\right)$ converges uniformly for $k \in \mathbf{N}$. In particular, $b\left(x_{k} / 2^{k}, y_{k} / 2^{k}\right) \rightarrow 0$ contradicting (\#).

If in addition to the assumptions above the space $E$ is a braked space (i.e., whenever $x_{k} \rightarrow 0$ there exists a sequence $t_{k} \rightarrow \infty$ such that $t_{k} x_{k} \rightarrow 0$ ([Kh]); for example metric linear spaces are braked), $b$ is hypocontinuous in the sense that if $x_{k} \rightarrow 0$ in $E$ and $B \subset F$ is bounded, then $b\left(x_{k}, y\right) \rightarrow 0$ uniformly for $y \in B$. To see this it suffices to show that $b\left(x_{k}, y_{k}\right) \rightarrow 0$ for $y_{k} \in B$. Now there exists $t_{k} \rightarrow \infty$ such that $t_{k} x_{k} \rightarrow 0$ and the result above 
implies that $\left\{b\left(t_{k} x_{k}, y_{k}\right)\right\}$ is bounded so $\frac{1}{t_{k}} b\left(t_{k} x_{k}, y_{k}\right)=b\left(x_{k}, y_{k}\right) \rightarrow 0$ as desired. This implies a result similar to that of Mazur and Orlicz. Namely, if $E$ is braked and sequentially complete, then $b$ is jointly sequentially continuous.

We finally consider a uniform boundedness result for bilinear mappings.

Case 32. Let $E, F, G$ be locally convex spaces with $E, F$ sequentially complete and $E$ braked. Let $\Gamma$ be a family of separately continuous bilinear mappings from $E \times F$ into $G$ which is pointwise bounded on $E \times F$. If $A \subset E, B \subset F$ are bounded, we claim that $\Gamma(A, B)$ is bounded, i.e., $\Gamma$ is uniformly bounded on bounded subsets of $E \times F$. If the conclusion fails to hold, there exist a continuous seminorm $p$ on $G, x_{k} \in A, y_{k} \in B, b_{k} \in \Gamma$ such that

$$
\text { (*) } p\left(b_{k}\left(x_{k}, y_{k}\right)\right)>2^{2 k} \text {. }
$$

Consider the triple $\left(E \times F,\left\{b_{k}\right\}: G\right)$ under the map $\left((x, y), b_{k}\right) \rightarrow b_{k}(x, y)$. The series $\sum_{k}\left(x_{k}, y_{k}\right) / 2^{k}$ is absolutely convergent in $E \times F$ and, therefore, subseries convergent by the sequential completeness assumption. By the remarks following Case 31, each $b_{k}$ is jointly sequentially continuous so the series is $w\left(E \times F,\left\{b_{k}\right\}\right)$ subseries convergent. Also, by the pointwise boundedness assumption the sequence $b_{k} / 2^{k} \rightarrow 0$ in $w\left(\left\{b_{k}\right\}, E \times F\right)$ so the sequence is relatively $w\left(\left\{b_{k}\right\}, E \times F\right)$ compact. By Theorem 10 the series $\sum_{j=1}^{\infty} b_{k}\left(x_{j} / 2^{j}, y_{j}\right) / 2^{k}$ converge uniformly for $k \in \mathbf{N}$. In particular, $b_{k}\left(x_{k}, y_{k}\right) / 2^{2 k} \rightarrow 0$ contradicting $\left(^{*}\right)$. This result also implies that $\Gamma$ is left sequentially equihypocontinuous with respect to the bounded subsets of $F$. For suppose $x_{k} \rightarrow 0$ in $E$ and $B \subset F$ is bounded in $F$ and $t_{k} \rightarrow \infty$ with $t_{k} x_{k} \rightarrow 0$. Let $p$ be a continuous seminorm on $G$ and set $M=$ $\sup \left\{p\left(b\left(t_{k} x_{k}, y\right): k \in \mathbf{N}, y \in B, b \in \Gamma\right\} ; M<\infty\right.$ by the result above. Then

$$
p\left(b\left(x_{k}, y\right)\right)=\frac{1}{t_{k}} p\left(b\left(t_{k} x_{k}, y\right)\right) \leq M / t_{k} \rightarrow 0
$$

uniformly for $b \in \Gamma$. In particular, this implies that $\Gamma$ is sequentially equicontinuous.

A similar uniform boundedness result for bilinear maps is given in 6.3.1 of [Sw5].

\section{Partial Sums}


We consider boundedness for the partial sums of a subseries convergent series. Let $\sum x_{j}$ be a $w(E, F)$ subseries convergent series. The partial sums of the series is defined to be

$$
\mathcal{S}=\left\{\sum_{j \in \sigma} x_{j}: \sigma \subset \mathbf{N}\right\}
$$

We first consider compactness for $\mathcal{S}$. Recall that a subseries convergent series is also unordered convergent in the sense that for any neighborhood of $0, U$, there exists $N$ such that $\sum_{j \in \sigma} x_{j} \in U$ whenever min $\sigma \geq N$ (see $[\mathrm{R}]$ or the conclusion of Theorem 10).

Lemma 33. Let $\Omega=\{0,1\}$ and define $\varphi: \Omega^{\mathbf{N}} \rightarrow E$ by $\varphi\left(\left\{t_{j}\right\}\right)=\sum_{j \in \sigma} x_{j}$, where $\sigma=\left\{j: t_{j}=1\right\}$. Then $\varphi$ is continuous when $\Omega^{\mathbf{N}}$ has the product topology and $E$ has $w(E, F)$.

Proof. Let $\left\{t^{\delta}\right\}$ be a net in $\Omega^{\mathbf{N}}$ which converges to $t=\left\{t_{j}\right\} \in \Omega^{\mathbf{N}}$. If $\left\{t_{j}^{\delta}\right\}=t^{\delta}$ and $t=\left\{t_{j}\right\}$, then $t_{j}^{\delta} \rightarrow t_{j}$ for every $j$ so $t_{j}^{\delta}=t_{j}$ eventually. Let $U$ be a neighborhood of 0 in $G$ and pick a symmetric neighborhood of $0, V$, such that $V+V \subset U$. Let $y \in F$ and set $\sigma=\left\{j: t_{j}=1\right\}, \sigma^{\delta}=\left\{j: t_{j}^{\delta}=1\right\}$ and $\sigma(n)=\{j \in \sigma: j \geq n\}$. By the unordered convergence of $\sum x_{j}$ there exists $n$ such that $\sum_{j \in \sigma(n)} x_{j} \in V, \sum_{j \in \sigma^{\delta}(n)} x_{j} \in V$ for all $\delta$. There exists $\delta_{0}$ such that $\delta \geq \delta_{0}$ implies $t_{j}^{\delta}=t_{j}$ for $1 \leq j \leq n$. Hence, if $\delta \geq \delta_{0}$, then

$$
\varphi\left(\left\{t_{j}^{\delta}\right\}\right) \cdot y-\varphi\left(\left\{t_{j}\right\}\right) \cdot y=\sum_{j \in \sigma^{\delta}(n)} x_{j} \cdot y-\sum_{j \in \sigma(n)} x_{j} \cdot y \in V+V \subset U .
$$

so $\varphi\left(t^{\delta}\right) \rightarrow \varphi(t)$ in $w(E, F)$.

Since $\Omega^{\mathbf{N}}$ is compact, sequentially compact and countably compact with respect to the product topology, Lemma 33 gives

Theorem 34. $\mathcal{S}$ is compact, sequentially compact and countably compact with respect to $w(E, F)$.

In particular, the set of partial sums of a subseries convergent series in an Abelian topological group is compact, sequentially compact and countably compact $([\mathrm{R}])$.

We next consider the boundedness of the partial sums in a semiconvex topological vector space. A subset $U$ of a topological vector space is semiconvex if there exists $a>0$ such that $U+U \subset a U$; for example, if $U$ is convex we may take $a=2$. A topological vector space $G$ is semiconvex if it has a neighborhood base of semiconvex subsets $([\mathrm{R}])$. The spaces $l^{p}$ $(0<p<1)$ are semiconvex but not locally convex. 
Theorem 35. Let $G$ be a semiconvex space and let $B \subset F$ be pointwise bounded on $E$, i.e., $\{x \cdot y: y \in B\}$ is bounded in $G$ for every $x \in E$. Then $\{x \cdot y: x \in \mathcal{S}, y \in B\}$ is bounded, i.e., $B$ is uniformly bounded on $\mathcal{S}$.

Proof. First, note that if $\sigma \subset \mathbf{N}$ satisfies the condition that the set $E_{\sigma}=\left\{\sum_{j \in \tau} x_{j}: \tau \subset \sigma\right\}$ is not absorbed by the semiconvex neighborhood $U$ of $G$ and if $V$ is a symmetric neighborhood such that $V+V \subset U$, then for every $k \in \mathbf{N}$ there exists a partition $\left(\alpha^{k}, \beta^{k}\right)$ of $\sigma, n_{k}>k$ and $y_{k} \in B$ such that $\sum_{j \in \alpha^{k}} x_{j} \cdot y_{k} \notin n_{k} V, \sum_{j \in \beta^{k}} x_{j} \cdot y_{k} \notin n_{k} V$. [By the bounded hypothesis for each $x=\sum_{j \in \sigma} x_{j} \in \mathcal{S}$, there is an $n_{k} \geq k$ such that $\left\{\sum_{j \in \sigma} x_{j} \cdot y: y \in B\right\} \subset n_{k} V$. But, $E_{\sigma} n_{k}(V+V)$ since $V+V \subset U$. So there exist $\alpha^{k} \subset \sigma, y_{k} \in B$ such that $\sum_{j \in \alpha^{k}} x_{j} \cdot y_{k} \notin n_{k}(V+V)$ and, hence, $\sum_{j \in \alpha^{k}} x_{j} \cdot y_{k} \notin n_{k} V$. If $\beta^{k}=\sigma \backslash \alpha^{k}$, then $\sum_{j \in \beta^{k}} x_{j} \cdot y_{k} \notin n_{k} V$ because otherwise

$$
\left.\sum_{j \in \alpha^{k}} x_{j} \cdot y_{k}=\sum_{j \in \sigma} x_{j} \cdot y_{k}-\sum_{j \in \beta^{k}} x_{j} \cdot y_{k} \in n_{k} V+n_{k} V \subset n_{k}(V+V) .\right]
$$

If the conclusion fails, there exists a semiconvex neighborhood $U$ of 0 which does not absorb $E_{\mathbf{N}}$. Let $V$ be a closed, symmetric neighborhood such that $V+V \subset U$. By the observation above there exists a partition $\left(\alpha^{1}, \beta^{1}\right)$ of $\sigma^{1}=\mathbf{N}, n_{1}$ and $y_{1} \in B$ such that $\sum_{j \in \alpha^{1}} x_{j} \cdot y_{1} \notin n_{1} V, \sum_{j \in \beta^{1}} x_{j}$. $y_{1} \notin n_{1} V$. Either $E_{\alpha^{1}}$ or $E_{\beta^{1}}$ is not absorbed by $U$ [ if both are absorbed by $U$, there is $m$ such that $E_{\alpha^{1}}+E_{\beta^{1}}=E_{\sigma^{1}} \subset m(U+U) \subset m(a U)$; this is where semiconvexity is used ]; pick whichever of $\alpha^{1}$ or $\beta^{1}$ satisfies this condition and label it $A^{1}$ and set $B^{1}=\sigma^{1} \backslash A^{1}$. Now treat $A^{1}$ as above and obtain a partition $\left(A^{2}, B^{2}\right)$ of $A^{1}, n_{2}>n_{1}, y_{2} \in B$ such that $\sum_{j \in A^{2}} x_{j} \cdot y_{2} \notin n_{2} V, \sum_{j \in B^{2}} x_{j} \cdot y_{2} \notin n_{2} V$. and $E_{A^{2}}$ is not absorbed by $U$. Continuing this construction produces a pairwise disjoint sequence $\left\{B_{k}\right\}$ of subsets of $\mathbf{N}$, increasing $\left\{n_{k}\right\}$ and $y_{k} \in B$ such that

$$
\text { (\#) } \sum_{j \in B^{k}} x_{j} \cdot y_{k} \notin n_{k} V,
$$

and since $V$ is closed we may assume that each $B^{k}$ is finite.

Now consider the matrix

$$
M=\left[m_{i j}\right]=\left[\frac{1}{n_{i}} \sum_{l \in B^{j}} x_{l} \cdot y_{i}\right] .
$$

We claim that $M$ is a $\mathcal{K}$ matrix. First, the columns of $M$ converge to 0 since $B$ is pointwise bounded on $E$. Suppose $\left\{k_{j}\right\}$ is an increasing sequence 
of integers and set $\tau=\cup_{j=1}^{\infty} B^{k_{j}}$. Since the $\left\{B^{j}\right\}$ are pairwise disjoint and finite and $\sum x_{j}$ is $w(E, F)$ subseries convergent, we have

$$
\sum_{j=1}^{\infty} \frac{1}{n_{i}} \sum_{l \in B^{k_{j}}} x_{l} \cdot y_{i}=\frac{1}{n_{i}} \sum_{l \in \tau} x_{l} \cdot y_{i}=\frac{1}{n_{i}} x_{\tau} \cdot y_{i} \rightarrow 0 .
$$

Hence, $M$ is a $\mathcal{K}$ matrix and by the Antosik-Mikusinski Matrix Theorem the diagonal of $M$ converges to 0 . But this contradicts (\#).

We will make a remark on the semiconvexity assumption following Corollary 37.

Corollary 36. Let $E$ be a semiconvex space with a nontrivial dual $E^{\prime}$. If $\sum x_{j}$ is $\sigma\left(E, E^{\prime}\right)$ subseries convergent, then the set of partial sums, $\mathcal{S}$, of $\sum x_{j}$ is $\beta\left(E, E^{\prime}\right)$ bounded. In particular, this holds for locally convex spaces.

Example 15 shows that a series may be weak subseries convergent but fail to be subseries convergent in the strong topology while Corollary 36 shows that the partial sums are always bounded in the strong topology.

We now observe that the Nikodym Boundedness Theorem follows from Theorem 35.

Corollary 37. (Nikodym) Let $\Sigma$ be a $\sigma$ algebra of subsets of a set $S, G$ be a semiconvex space. If $\mathcal{M}$ is a family of countably additive $G$ valued set functions defined on $\Sigma$ which is pointwise bounded on $\Sigma$, then $\mathcal{M}$ is uniformly bounded on $\Sigma$, i.e., $\{\mu(A): \mu \in \mathcal{M}, A \in \Sigma\}$ is bounded.

Proof. The proof of Theorem 35 shows that if $\{\mu(A): \mu \in \mathcal{M}, A \in \Sigma\}$ is not bounded, there exist $\left\{\mu_{k}\right\} \subset \mathcal{M}$ and a pairwise disjoint sequence $\left\{B_{k}\right\} \subset \Sigma$ such that $\left\{\mu_{k}\left(B_{k}\right)\right\}$ is not bounded [this is a generalization of a well known result of Drewnowski $([\mathrm{Dr}])]$. In the notation of Example 3 the series $\sum B_{k}$ is $w(\Sigma, \mathcal{M})$ subseries convergent and $\mathcal{M}$ is pointwise bounded on $\mathcal{S}=\left\{\cup_{j \in \sigma} B_{j}: \sigma \subset \mathbf{N}\right\}$ so $\mathcal{M}$ is uniformly bounded on $\mathcal{S}$ by Theorem 35. In particular, $\left\{\mu_{k}\left(B_{k}\right)\right\}$ is bounded.

Corollary 37 is applicable to the case when $\mathcal{M}$ is single measure so a countably additive set function with values in a semiconvex space has bounded range; this gives a generalization of 3.6.3 of [Rol]. Turpin has given an example of a countably additive set function defined on a $\sigma$ algebra with values in a nonlocally convex space which has unbounded range so the semiconvex assumption cannot be dropped ([Rol]3.6.4). What conditions 
on the space which are necessary and sufficient for a vector measure to have bounded range seem to be unknown.

The version of the Nikodym Boundedness Theorem for semiconvex spaces is due to Contantinescu ([Co]) and Weber ([We]).

\section{References}

[AB] P. Antosik, Z. Bu, R. Li, E. Pap and C. Swartz, $\mathcal{K}$-convergent sequences in locally convex spaces, Generalized Functions and Convergence, World Scientific, Singapore, pp. 299-304, (1990).

[AS1] P. Antosik and C. Swartz, Matrix Methods in Analysis, SingerVerlag, Heidelberg, (1985).

[AS2] P. Antosik and C. Swartz, The Schur and Phillips Lemmas for Topological Groups, J. Math. Anal Appl., 98, pp. 179-187, (1984).

[B] B. Basit, On a Theorem of Gelfand and a new proof of the OrliczPettis Theorem, Rend. Inst. Matem. Univ. di Trieste, 18 (1986), pp. 159-162, (1986).

[BK] G. Bennett and N. Kalton, FK-spaces containing $c_{0}$, Duke Math. J., 39, pp. 561-582, (1972).

[C] C. Constantinescu, On Nikodyms Boundedness Theorem, Libertas Math., 1, pp. 51-73, (1981).

[BCS] O. Blasco, J. M. Calabuig and T. Signes, A bilinear version of OrliczPettis Theorem, J. Math. Anal. Appl., 348, pp. 150-164, (2008).

[CL] A. Chen and R. Li, A version of Orlicz-Pettis Theorem for quasihomogeneous operator space, J. Math. Anal. Appl., 373, pp. 127-133, (2011).

[Diel] P. Dierolf, Theorems of Orlicz-Pettis type for locally convex spaces, Man. Math., 20, pp. 73-94, (1977).

[DF] J. Diestel and B. Faires, On Vector Measures, Trans. Amer. Math. Soc., 198, pp. 253-271, (1974). 
[DU] J. Diestel and J. Uhl, Vector Measures, Amer. Math. Soc. Surveys \#15, Providence, (1977).

[Di] N. Dinculeanu, Weak Compactness and Uniform Convergence of Operators in Spaces of Bochner Integrable Function, J. Math. Anal. Appl., 109 (1985).

[Dr] L. Drewnowski, Equivalence of Brooks-Jewett, Vitali-Hahn-Saks and Nikodym Theorems, Bull. Aced. Polon. Sci., 20, pp. 725-731, (1972).

[DS] N. Dunford and J. Schwartz, Linear Operators, Interscience, N.Y., (1958).

[G] H. Garnir, M. DeWilde and J. Schmets, Analyse Fontionnelle I, Birkhauser, Basel, (1968).

[FL] W. Filter and I. Labuda, Essays on the Orlicz Theeorem I, Real Anal. Exch., 16, pp. 393-403, (1990-1991).

[K2] N. Kalton, Subseries Convergence in Topological Groups and Vector Spaces, Israel J. Math., 10, pp. 402-412, (1971).

[K3] N. Kalton, Spaces of Compact Operators, Math. Ann., 208, pp. 267278, (1974).

[Kh] S. M. Khaleelulla, Counter Examples in Topological Vector Spaces, Springer Lecture Notes in Mathematics 936, Heidelberg, (1982).

[LC] R.Li and M. Cho, A General Kalton-type Theorem, J. Harbin Inst. Tech 25, pp. 100-104, (1992).

[LS1] R. Li and C. Swartz, Spaces for which the Uniform Boundedness Principle Holds, Studia Sci. Math. Hungar., 27, pp. 379-384, (1992).

[LS2] R. Li and C. Swartz, A Nonlinear Schur Theorem, Acta Sci. Math. (Szged), 58, pp. 497-508, (1993).

[LS3] R. Li and C. Swartz, An Abstract Orlicz-Pettis Theorem and Applications, Proy. J. Math., 27, pp. 155-169, (2008).

[LT] J. Lindenstrauss and L. Tzarfriri, Classical Banach Spaces I, SpringerVerlag, Berlin, (1977). 
[LW] R. Li and J. Wang, Invariants in Abstract Mappping Pairs, J. Aust. Math. Soc., 76, pp. 369-381, (2004).

[MO] S. Mazur and W. Orlicz, Uber Folgen linearen Operationen, Studia Math., 4, pp. 152-157, (1933).

[O] W. Orlicz, Beitrage zur Theorie der Orthogonalent Wichlungen II, Studia Math., 1, pp. 241-255, (1929).

[P] B. Pettis, On Integration in Vector Spaces, Trans. Amer. Math. Soc., 49, pp. 277-304, (1938).

[Pi] A. Pietsch, Nukleare Lokalconvexe Raume, Akademie Verlag, Berlin, (1965).

[MO] A. Mohsen, Weak*-Norm Sequentially Continuous Operators, Math. Slovaca, 50, pp. 357-363, (2000).

[R] A. Robertson, On unconditional convergence in topological vector spaces, Proc. Roy. Soc. Edinburgh, 68, pp. 145-157, (1969).

[Rol] S. Rolewicz, Metric Linear Spaces, Polish Sci. Publishers, Warsaw, (1984).

[St] W. Stiles, On subseries convergence in F-spaces, Israel J. Math., 8, pp. 53-56, (1970).

[Sw1] C. Swartz, An Introduction to Functional Analysis, Dekker, N.Y., (1992).

[Sw2] C. Swartz. A Generalized Orlicz-Pettis Theorem and Applications, Math. Z., 163, pp. 283-290, (1978).

[Sw3] C. Swartz, An Abstract Orlicz-Pettis Theorem, Bull. Pol. Acad. Sci. 32, pp. 433-437, (1984).

[Sw4] C. Swartz, A Generalization of Stiles Orlicz-Pettis Theorem, Rend. Inst. Matem Univ. di Trieste, 20, pp. 109-112, (1988).

[Sw5] C. Swartz, Infinite Matrices and the Gliding Hump, World Sci. Publ., Singapore, (1996).

[Sw6] C. Swartz, Multiplier Convergent Series, World Sci. Publ., Singapore, (2009). 
[Sw7] C. Swartz. A Bilinear Orlicz-Pettis, Theorem, J. Math. Anal. Appl., 365, pp. 332-337, (2010).

[Th] G. Thomas, L'integration par rapport a une mesure de Radon vectorielle, Ann. Inst. Fourier, 20, pp. 55-191, (1970).

[Tw] I. Tweddle, Unconditional Convergence and Vector-Valued Measures, J. London Math. Soc., 2, pp. 603-610, (1970).

[We] H. Weber, Compactness in Spaces of Group-Valued Contents, the Vitali-Hahn-Saks Theorem and Nikodym Boundedness Theorem, Rocky. Mount. J. Math., 16, pp. 253-275, (1985).

[Wi] A. Wilansky, Modern Methods in Topological Vector Spaces, Mc Graw-Hill, N. Y. (1978).

[ZCL] F. Zheng, C. Cui, and R. Li, Abstract Gliding Hump Properties in the Vector-Valued Dual Pairs, Acta Anal. Functionalis Appl., 12, pp. 322-327, (2010).

\section{Min-Hyung Cho}

Department of Mathematics

Kum-Oh National Institute of Technology

Kumi, Korea

e-mail : mignon@kumoh.ac.kr

\section{Li Ronglu}

Department of Mathematics

Harbin Institute of Technology

Harbin,

P. R. C.

and

\section{Charles Swartz}

Department of Mathematics

New Mexico State University

Las Cruces, NM, 88003,

U. S. A.

e-mail : cswartz@nmsu.edu 